\title{
Quantum secure direct communication without using perfect quantum channel
}

\author{
Jian Wang * Quan Zhang, and Chao-jing Tang \\ School of Electronic Science and Engineering, \\ National University of Defense Technology, \\ Changsha, 410073, China
}

\begin{abstract}
Most of the quantum secure direct communication protocol needs a pre-established secure quantum channel. Only after insuring the security of quantum channel, could the sender encode the secret message and send them to the receiver through the secure channel. In this paper, we present a quantum secure direct communication protocol using Einstein-Podolsky-Rosen pairs. It is not necessary for the present protocol to insure the security of quantum channel before transmitting the secret message. In the present protocol, all Einstein-Podolsky-Rosen pairs are used to transmit the secret message except those chosen for eavesdropping check.
\end{abstract}

PACS numbers: 03.67.Dd, 03.65.Ud, 42.79.Sz

\section{INTRODUCTION}

Quantum key distribution (QKD) is one of the most promising applications of quantum information science. The goal of QKD is to allow two legitimate parties, Alice and Bob, to generate a secret key over a long distance, in the presence of an eavesdropper, Eve, who interferes with the signals. Since Bennett and Brassard presented the original QKD protocol [1], it has been developed quickly. Recently, a novel concept, quantum secure direct communication (QSDC) has been proposed [2]. Different from QKD whose object is to establish a common key between the communication parties, QSDC's object is to transmit the secret messages directly without first establishing a key to encrypt them. In this paper, we follow the above definition of QSDC.

QSDC can be used in some special environments which has been shown by Boströem and Deng et al. [3, 4]. The works on QSDC attracted a great deal of attentions [28]. We can divide these works into two kinds, one utilizes entangled state [3, 4, 6, 7, 8], the other utilizes singphoton [5]. Boström and Felbinger proposed a Ping-Pong QSDC protocol which is quasi-secure for secure direct communication if perfect quantum channel is used [3]. Deng et al. put forward a two-step QSDC protocol using Einstein-Podolsky-Rosen (EPR) pairs (hereafter called Deng's protocol) [4] and a QSDC scheme with a quantum one-time pad [5]. Feng Li Yan and Xiao Qiang Zhang presented a QSDC scheme using EPR pairs and teleportation (hereafter called Yan's scheme) [6]. The security of most of QSDC schemes relies on a pre-established secure quantum channel. Deng et al. pointed out the basic requirement for QSDC scheme in [5], which eavesdropping check before the message being encoded must be performed first. Only in this way, can the sender utilize the checked channel to encode the secret message and transmit it to the receiver.

*Electronic address: jwang@nudt.edu.cn
Actually, the communication parties, Alice and Bob can obtain a common key as long as a secure quantum channel is established. Alice can then send her secret messages to Bob directly by classical channel. For example, in Yan's scheme [6], Alice and Bob can obtain a common key as long as they measure their particles in the $Z$-basis. It is not necessary for the sender to transmit her secret to the receiver using teleportation after insuring the security of quantum channel. Note that it needs two-bit classical information to recovery one bit secret message in Yan's scheme. It would be better to transmit directly the secret to the receiver using classical channel. Suppose the secret messages to be transmitted is 101001 and the common key between Alice and Bob is 001101. Alice tells Bob to reorder his results of measurements by choosing his result of the third, the first, the fourth, the second, the fifth, and the sixth particle in turn. That is to say Bob can recover Alice's secret messages 101001 as long as he reorders his results of measurements according Alice's classical messages and the eavesdropper, Eve cannot obtain any information about the secret.

In this paper, we present a QSDC protocol with EPR pairs without insuring the security of quantum channel before transmitting the secret message. The secret message is deterministically sent through the quantum channel. We also show the present protocol is secure and efficient.

This paper is organized as follows. In Sec III we describe the process of the QSDC protocol. In Sec III we discuss the security and efficiency of the present protocol. Finally, we give a summary in Sec IV

\section{THE QSDC PROTOCOL}

In the QSDC protocol, we suppose the sender Alice wants to send a secret message to the receiver, say Bob directly. The basic idea of the protocol originates from quantum teleportation $[9]$. Alice entangles his encoded secret message state with prepared EPR pairs. She then performs controlled-NOT (CNOT) operation and Hadamard transformation, which is similar 
to the method used in quantum teleportation. Yan's scheme utilized the method of quantum teleportation faithfully. After insuring the security of quantum channel, the receiver performs unitary operation on his particle to recover the sender's secret message according to the sender's classical message. The basic idea of our protocol is different from Yan's scheme. Instead of performing unitary operation to recover Alice's secret message, Bob measures his particle in a fixed measuring basis and recovers Alice's secret message according to the correlation between two parties' results. The QSDC protocol is as follows:

(1)Alice prepares an ordered $N$ EPR pairs in the state

$$
|\phi\rangle_{A B}=\frac{1}{\sqrt{2}}(|00\rangle+|11\rangle)_{A B} .
$$

We denotes the ordered $N$ EPR pairs with $\left\{\left[\mathrm{P}_{1}(A), \mathrm{P}_{1}(B)\right], \quad\left[\mathrm{P}_{2}(A), \mathrm{P}_{2}(B)\right], \cdots, \quad\left[\mathrm{P}_{N}(A), \mathrm{P}_{N}(B)\right]\right\}$, where the subscript indicates the pair order in the sequence, and $A, B$ represents the two particles of each EPR pair, respectively. Alice takes one particle from each EPR pair to form an ordered EPR partner particle sequence $\left[\mathrm{P}_{1}(A), \mathrm{P}_{2}(A), \cdots, \mathrm{P}_{N}(A)\right]$, called $A$ sequence. The remaining EPR partner particles compose $B$ sequence, $\left[\mathrm{P}_{1}(B), \mathrm{P}_{2}(B), \cdots, \mathrm{P}_{N}(B)\right]$. Alice transmits $B$ sequence to Bob.

(2) Bob selects randomly a sufficiently large subset of $B$ sequence and performs Hadamard transformations

$$
H=\frac{1}{\sqrt{2}}\left(\begin{array}{cc}
1 & 1 \\
1 & -1
\end{array}\right)
$$

on them. He then announces publicly the position of the selected particles. The Hadamard transformation is crucial for the security of the protocol as we will see in the sequel.

(3) After hearing from Bob, Alice executes Hadamard transformations on the corresponding particles of $A$ sequence. She then selects randomly a sufficiently large subset of particles from $A$ sequence, which we call $C$ sequence. Alice generates a random bit string and encodes it on $C$ sequence. If Alice's random bit is "0" (" 1 "), she prepares a particle $a$ in the state $|+\rangle=\frac{1}{\sqrt{2}}(|0\rangle+|1\rangle)$ $\left(|-\rangle=\frac{1}{\sqrt{2}}(|0\rangle-|1\rangle)\right)$ for each particle of $C$ sequence. $C$ sequence is used to check eavesdropping, which we call checking sequence. The remaining particles of $A$ sequence forms $D$ sequence. $D$ sequence is used to encode Alice's secret message, which we call encoding sequence. Alice then encodes her secret message on $D$ sequence. If Alice's secret message is " 0 " ( " 1 "), she prepares a particle $a$ in the state $|+\rangle(|-\rangle)$ for each particle of $D$ sequence. Thus Alice prepares $N$ particles for each particle of $A$ sequence, which we call $a$ sequence $\left[\mathrm{P}_{1}(a), \mathrm{P}_{2}(a), \cdots\right.$, $\left.\mathrm{P}_{N}(a)\right]$.

(4) If the state of the particle $\mathrm{P}_{i}(a)$ is $|+\rangle$, then the state of the particle $\mathrm{P}_{i}(a), \mathrm{P}_{i}(A)$, and $\mathrm{P}_{i}(B)(i=$ $1,2, \cdots, N)$ is

$$
\left|\Phi_{0}\right\rangle_{a A B}=\frac{1}{\sqrt{2}}(|0\rangle+|1\rangle)_{a} \otimes \frac{1}{\sqrt{2}}(|00\rangle+|11\rangle)_{A B},
$$

where the subscript $a$ denotes the particle $\mathrm{P}_{i}(a)$. If the state of the particle $\mathrm{P}_{i}(a)$ is $|-\rangle$, then the state of the particle $\mathrm{P}_{i}(a), \mathrm{P}_{i}(A)$, and $\mathrm{P}_{i}(B)$ is

$$
\left|\Phi_{1}\right\rangle_{a A B}=\frac{1}{\sqrt{2}}(|0\rangle-|1\rangle)_{a} \otimes \frac{1}{\sqrt{2}}(|00\rangle+|11\rangle)_{A B} .
$$

(5) Alice sends the particle $\mathrm{P}_{i}(a), \mathrm{P}_{i}(A) \quad(i=$ $1,2, \cdots, N)$ through a CNOT gate (the particle $\mathrm{P}_{i}(a)$ is the controller, the particle $\mathrm{P}_{i}(A)$ is the target). Then $\left|\Phi_{0}\right\rangle_{a A B}$ is changed to

$$
\left|\Phi_{0}^{\prime}\right\rangle_{a A B}=\frac{1}{2}(|000\rangle+|110\rangle+|011\rangle+|101\rangle)_{a A B},
$$

and $\left|\Phi_{1}\right\rangle_{a A B}$ becomes

$$
\left|\Phi_{1}^{\prime}\right\rangle_{a A B}=\frac{1}{2}(|000\rangle-|1100\rangle+|0111\rangle-|101\rangle)_{a A B} .
$$

(6) Alice performs Hadamard transformation on the particle $\mathrm{P}_{i}(a)$, obtaining

$$
\begin{aligned}
\left|\Phi_{0}^{\prime \prime}\right\rangle_{a A B}= & \frac{1}{2}\left[|00\rangle_{a A} \otimes \frac{1}{\sqrt{2}}(|0\rangle+|1\rangle)_{B}\right. \\
& +|10\rangle_{a A} \otimes \frac{1}{\sqrt{2}}(|0\rangle-|1\rangle)_{B} \\
& +|01\rangle_{a A} \otimes \frac{1}{\sqrt{2}}(|0\rangle+|1\rangle)_{B} \\
& \left.+|11\rangle_{a A} \otimes \frac{1}{\sqrt{2}}(|1\rangle-|0\rangle)_{B}\right]
\end{aligned}
$$

or

$$
\begin{aligned}
\left|\Phi_{1}^{\prime \prime}\right\rangle_{a A B}= & \frac{1}{2}\left[|00\rangle_{a A} \otimes \frac{1}{\sqrt{2}}(|0\rangle-|1\rangle)_{B}\right. \\
& +|10\rangle_{a A} \otimes \frac{1}{\sqrt{2}}(|0\rangle+|1\rangle)_{B} \\
& +|01\rangle_{a A} \otimes \frac{1}{\sqrt{2}}(|1\rangle-|0\rangle)_{B} \\
& \left.+|11\rangle_{a A} \otimes \frac{1}{\sqrt{2}}(|0\rangle+|1\rangle)_{B}\right] .
\end{aligned}
$$

(7) Alice then measures the particle $\mathrm{P}_{i}(a), \mathrm{P}_{i}(A)$ in the $Z$-basis, $\{|0\rangle,|1\rangle\}$. Bob measures the particle $\mathrm{P}_{i}(B)$ in the $X$-basis, $\{|+\rangle,|-\rangle\}$. At this step, although Bob obtains his result of measurement, he cannot recover Alice's secret message without Alice's result. We can draw the above conclusion according to the equation 7 and 8

(8) Alice informs Bob the positions of $C$ sequence (checking sequence) and lets him announce his corresponding results of measurements. Alice judges whether her random bits can be reconstructed correctly by combining Bob's results and her results of $C$ sequence. If the error rate is small, Alice can conclude that there is no eavesdroppers in the line. Alice and Bob continue to perform the next step, otherwise they abort the communication. 
TABLE I: The recovery of Alice's secret message

\begin{tabular}{|c|c|c|}
\hline Alice's result & Bob's result & secret message \\
\hline 0 & $|+\rangle$ & 0 \\
\hline 0 & $|-\rangle$ & 1 \\
\hline 1 & $|+\rangle$ & 1 \\
\hline 1 & $|-\rangle$ & 0 \\
\hline
\end{tabular}

(9) If Alice is certain that there is no eavesdropping, she announces the results of measurements of $D$ sequence. Thus Bob can recover Alice's secret message, according to Alice's result, as illustrated in Table 1.

Suppose Bob's result is $|+\rangle$. If Alice's result of measurement of the corresponding $D$ sequence particle is " 0 " ("1"), they then conclude that the Alice's secret message is " 0 " (" 1 ").

\section{SECURITY AND EFFICIENCY OF THE QSDC PROTOCOL}

So far we have proposed the QSDC protocol. We now discuss the security of the present protocol. The crucial point is that the Hadamard gate at the step 2 and 3 of the scheme do not allow an eavesdropper, Eve to have a successful attack and Eve's attack will be detected during the eavesdropping check.

We first consider the intercept-resend attack strategy. In this attack strategy, Eve intercepts the particles of $B$ sequence transmitted to Bob and makes measurements on them. Then she resends a particle sequence to Bob according to her results of measurements. Eve can only intercept $B$ sequence at the step 1 of the protocol and she cannot make certain which particle will be executed Hadamard transformation. Suppose Eve measures the intercepted particle on which Alice and Bob will not perform Hadamard transformation in the $Z$-basis. In this way, if the result of Eve's measurement is " 0 ", she sends a particle in the state $|+\rangle$ to Bob, otherwise sends a particle in the state $|-\rangle$. Then if Alice prepares a particle, $\mathrm{P}_{i}(a)$ in the state $|+\rangle$, the state of $\left[\mathrm{P}_{i}(a), \mathrm{P}_{i}(A), \mathrm{P}_{i}(B)\right]$ collapses to $|+0+\rangle_{a A B}$ or $|+1-\rangle_{a A B}$ each with probability $1 / 2$. After Alice's CNOT operation and Hadamard transformation, the state becomes

$$
\left|\Psi_{i}\right\rangle_{a A B}=\frac{1}{2}(|00\rangle+|01\rangle+|10\rangle-|11\rangle)_{a A} \otimes|+\rangle_{B}
$$

or

$$
\left|\Psi_{i}\right\rangle_{a A B}=\frac{1}{2}(|00\rangle+|01\rangle-|10\rangle+|11\rangle)_{a A} \otimes|-\rangle_{B} .
$$

Alice measures $\mathrm{P}_{i}(a)$ in the $Z$-basis and obtains " 0 " or "1", each with probability $1 / 2$. She has only $50 \%$ probability of obtaining the right result. During the eavesdropping check, Eve's attack will be detected easily. Similarly, If Alice prepares a particle, $\mathrm{P}_{i}(a)$ in the state $|-\rangle$, the error rate introduced by Eve will also achieve $50 \%$.
Suppose Eve measures the intercepted particle on which Alice and Bob will perform Hadamard transformation in the $X$-basis. Note that $|\phi\rangle_{A B}$ can also be expressed as $\frac{1}{\sqrt{2}}(|++\rangle+|--\rangle)_{A B}$. If Alice prepares a particle, $\mathrm{P}_{i}(a)$ in the state $|+\rangle$, then The state of $\left[\mathrm{P}_{i}(a)\right.$, $\left.\mathrm{P}_{i}(A), \mathrm{P}_{i}(B)\right]$ collapses to $|+++\rangle_{a A B}$ or $|+--\rangle_{a A B}$ each with probability $1 / 2$. After the Hadamard transformations of Alice and Bob, the state becomes $|+00\rangle_{a A B}$ or $|+11\rangle_{a A B}$. According to the protocol, the state is changed to

$$
\left|\Psi_{i}^{\prime}\right\rangle_{a A B}=\frac{1}{2 \sqrt{2}}(|0+\rangle+|1-\rangle)_{a A} \otimes(|+\rangle+|-\rangle)_{B}
$$

or

$$
\left|\Psi_{i}^{\prime}\right\rangle_{a A B}=\frac{1}{2 \sqrt{2}}(|0+\rangle-|1-\rangle)_{a A} \otimes(|+\rangle-|-\rangle)_{B}
$$

Obviously, Eve's eavesdropping will be detected during the eavesdropping check. It will have the same result if Alice prepares a particle, $\mathrm{P}_{i}(a)$ in the state $|-\rangle$.

We then consider the collective attack strategy. In this strategy, Eve intercepts the particle $\mathrm{P}_{i}(B)$ and uses it and her own ancillary particle in the state $|0\rangle$ to do a CNOT operation (the particle $\mathrm{P}_{i}(B)$ is the controller, Eve's ancillary particle is the target). Then Eve resends the particle $\mathrm{P}_{i}(B)$ to Bob. However, Eve cannot make certain which particle will be performed Hadamard transformation. Suppose Bob will not perform Hadamard transformation on the intercepted particle. The state of $\mathrm{P}_{i}(A)$, $\mathrm{P}_{i}(B)$, and Eve's corresponding ancillary particle is

$$
|\Omega\rangle_{A B E}=\frac{1}{\sqrt{2}}(|000\rangle+|111\rangle)_{A B E},
$$

where the subscript $E$ indicates Eve's ancillary particle. According to the protocol, the state of the particle $\mathrm{P}_{i}(a)$, $\mathrm{P}_{i}(A), \mathrm{P}_{i}(B)$, and the corresponding Eve's ancillary particle will be

$$
\begin{aligned}
\left|\Omega_{0}\right\rangle_{a A B E}= & \frac{1}{2}\left[|00\rangle_{a A} \otimes \frac{1}{\sqrt{2}}(|00\rangle+|11\rangle)_{B E}\right. \\
& +|10\rangle_{a A} \otimes \frac{1}{\sqrt{2}}(|00\rangle-|11\rangle)_{B E} \\
& +|01\rangle_{a A} \otimes \frac{1}{\sqrt{2}}(|00\rangle+|11\rangle)_{B E} \\
& \left.+|11\rangle_{a A} \otimes \frac{1}{\sqrt{2}}(|11\rangle-|00\rangle)_{B E}\right]
\end{aligned}
$$

or

$$
\begin{aligned}
\left|\Omega_{1}\right\rangle_{a A B E}= & \frac{1}{2}\left[|00\rangle_{a A} \otimes \frac{1}{\sqrt{2}}(|00\rangle-|11\rangle)_{B E}\right. \\
& +|10\rangle_{a A} \otimes \frac{1}{\sqrt{2}}(|00\rangle+|11\rangle)_{B E} \\
& +|01\rangle_{a A} \otimes \frac{1}{\sqrt{2}}(|11\rangle-|00\rangle)_{B E} \\
& \left.+|11\rangle_{a A} \otimes \frac{1}{\sqrt{2}}(|00\rangle+|11\rangle)_{B E}\right] .
\end{aligned}
$$


Note that

$$
\frac{1}{\sqrt{2}}(|00\rangle+|11\rangle)=\frac{1}{\sqrt{2}}(|++\rangle+|--\rangle)
$$

and

$$
\frac{1}{\sqrt{2}}(|00\rangle-|11\rangle)=\frac{1}{\sqrt{2}}(|+-\rangle+|-+\rangle) .
$$

We can rewritten $\left|\Omega_{0}\right\rangle_{a A B E}$ and $\left|\Omega_{1}\right\rangle_{a A B E}$ as

$$
\begin{aligned}
& \frac{1}{2}\left[|00\rangle_{a A} \otimes \frac{1}{\sqrt{2}}(|++\rangle+|--\rangle)_{B E}\right. \\
& +|10\rangle_{a A} \otimes \frac{1}{\sqrt{2}}(|+-\rangle-|-+\rangle)_{B E} \\
& +|01\rangle_{a A} \otimes \frac{1}{\sqrt{2}}(|++\rangle+|--\rangle)_{B E} \\
& \left.-|11\rangle_{a A} \otimes \frac{1}{\sqrt{2}}(|+-\rangle+|-+\rangle)_{B E}\right]
\end{aligned}
$$

and

$$
\begin{aligned}
& \frac{1}{2}\left[|00\rangle_{a A} \otimes \frac{1}{\sqrt{2}}(|+-\rangle+|-+\rangle)_{B E}\right. \\
& +|10\rangle_{a A} \otimes \frac{1}{\sqrt{2}}(|++\rangle-|--\rangle)_{B E} \\
& -|01\rangle_{a A} \otimes \frac{1}{\sqrt{2}}(|+-\rangle+|-+\rangle)_{B E} \\
& \left.+|11\rangle_{a A} \otimes \frac{1}{\sqrt{2}}(|++\rangle+|--\rangle)_{B E}\right]
\end{aligned}
$$

Alice then measures the particle $\mathrm{P}_{i}(a)$ in the $Z$-basis and Bob measures the particle $\mathrm{P}_{i}(B)$ in the $X$-basis. Suppose Alice's random bit is " 0 " and her result of measurement of the corresponding $C$ sequence particle is " 0 " ("1"). According to the protocol, Bob's result must be $|+\rangle(|-\rangle)$. Similarly, if Alice's random bit is "1" and her result of measurement of the corresponding $C$ sequence particle is " 0 " (" 1 "). Thus Bob's result must be $|-\rangle(|+\rangle)$. However, Bob can only obtains the right result with probability $1 / 2$ because of Eve's eavesdropping. During the eavesdropping check, half of Bob's results will be inconsistent with that of Alice's. Thus Eve's eavesdropping will be detected easily, because her eavesdropping introduces a error rate with $50 \%$. Eve measures the intercepted particle, but she can only obtain $|+\rangle$ or $|-\rangle$ each with probability $1 / 2$. Because she has no information about Alice's result, she cannot conclude what Alice's secret message is, even if she obtains Bob's result of measurement.
Suppose Eve execute Hadamard and CNOT operation on the intercepted particle which Bob will perform Hadamard transformation on it. Note that $|\phi\rangle_{A B}$ can be expressed as $\frac{1}{\sqrt{2}}(|++\rangle+|--\rangle)_{A B}$. Thus the state of $\mathrm{P}_{i}(A), \mathrm{P}_{i}(B)$ and Eve's corresponding ancillary particle will be

$$
\left|\Omega_{A B E}\right\rangle=\frac{1}{\sqrt{2}}(|++0\rangle+|--1\rangle)_{A B E} .
$$

After Hadamard transformation of Alice and Bob, $\left|\Omega_{A B E}\right\rangle$ is changed to $\frac{1}{\sqrt{2}}(|000\rangle+|111\rangle)_{A B E}$ which is equal to the equation 13. As we described above, Eve's eavesdropping can also be detected.

We now analyze the efficiency of the present protocol. In our protocol, all EPR pairs are used to transmit the secret message except those chosen for checking eavesdroppers because the measuring basis of communication parties is invariable. We only need to transmit particles once during the process of protocol, so we only need eavesdropping check once. Twice transmission of EPR particles and twice eavesdropping check are required in Deng's scheme.

\section{SUMMARY}

So far we have proposed a QSDC scheme using EPR pairs and analyzed the security and efficiency of the present protocol. To prevent eavesdropping, Alice and Bob perform Hadamard transformation on the randomly selected particles. Alice encodes her secret message into a given state and sends it to the receivers directly using quantum channel. Different from most of the QSDC schemes, it is not necessary for our protocol to insure the security of quantum channel before sending Alice's secret message. Without Alice's result of measurement, the receiver or the eavesdropper cannot have any information about Alice's secret message. Alice announces her result only if she is certain that there is no eavesdropping in the line and only in such a way can the receiver recover Alice's secret message. The present protocol is efficient in that all EPR pairs are used to transmit the secret message except those chosen for eavesdropping check.

\section{Acknowledgments}

This work is supported by the National Natural Science Foundation of China under Grant No. 60472032.
[1] C. H. Bennett and G. Brassard, in Proceedings of IEEE international Conference on Computers, Systems and signal Processing, Bangalore, India (IEEE, New York), pp.
175 - 179 (1984).

[2] A. Beige, B.-G. Englert, Ch. Kurtsiefer, and H. Weinfurter, Acta Phys. Pol. A 101, 357 (2002). 
[3] K. Boströem and T. Felbinger, Phys. Rev. Lett. 89, 187902 (2002).

[4] F. G. Deng, G. L. Long, and X. S. Liu, Phys. Rev. A 68, 042317 (2003).

[5] F. G. Deng and G. L. Long, Phys. Rev. A 69, 052319 (2004).

[6] F. L. Yan and X. Q. Zhang, Euro. Phys. J. B 41, 75 (2004).

[7] T. Gao, F. L. Yan, and Z. X. Wang, Chin. Phys. 14, 893
(2005).

[8] C. Wang, F. G. Deng, Y. S. Li, X. S. Liu, and G. L. Long, Phys. Rev. A 71, 044305 (2005).

[9] C. H. Bennett, G. Brassard, C. Crépeau, R. Jozsa, A. Peres, and W. K. Wootters, Phys. Rev. Lett. 70, 1895 (1993). 